\title{
Coronary age, based on coronary calcium measurement, is increased in patients with morbid obesity
}

\author{
Adam Lemanowicz ${ }^{\mathrm{A}, \mathrm{B}, \mathrm{D}, \mathrm{E}, \mathrm{F}}$, Marcin Białecki ${ }^{\mathrm{B}, \mathrm{E}}$, Waldemar Leszczyński ${ }^{\mathrm{C}}$, Mateusz Hawrył ${ }^{\mathrm{E}}$ \\ Department of Radiology and Diagnostic Imaging, Nicolaus Copernicus University in Toruń, Collegium Medicum in Bydgoszcz, Poland
}

\section{Abstract}

Purpose: Obesity is a well-known of risk factor for atherosclerosis. However, recently an "obesity paradox" has been discussed, which is considered as a protective effect of obesity on the development coronary artery disease (CAD). An aim of the study was to investigate the risk of CAD in morbidly obese patients using coronary artery calcium measurement.

Material and methods: Fifty-one patients with morbid obesity (BMI $\geq 40 \mathrm{~kg} / \mathrm{m}^{2}$ ) and thirty controls were scanned to determine the amount of coronary artery calcification (CAC), which was expressed as calcium score (CS) and coronary age (CA). The control group consisted of patients scanned for the clinical suspicion of CAD, who did not fulfil the criteria of obesity.

Results: Mean BMI of obese patients and controls was 47.3 and 26.5, respectively $(p<0.0001)$. Arterial hypertension, dyslipidaemia, and smoking were more frequent in the control group than in the obesity group $(p<0.02)$. The prevalence of CAC was higher in the obesity group ( $53 \%$ vs. $23 \%, p<0.01$ ). The groups did not differ regarding CS and CA. However, the difference between coronary age and metrical age was higher in obese patients than in controls ( +5.6 vs. -4.8 years, respectively, $p<0.005)$.

Conclusions: Patients with morbid obesity present an increased risk of CAD that is reflected by the difference between their coronary age and metrical age.

Key words: morbid obesity, coronary age, calcium score, atherosclerosis.

\section{Introduction}

There is a long-known adage, attributed to the famous English physician Thomas Sydenham (1624-1689): "you're only as old as your arteries". However, for many years only metrical age was used by clinicians trying to assess the cardiovascular risk of their patients via scales such as the Framingham scale. In this scale, used to calculate 10-year coronary risk, the age of patients is taken into account in addition to other factors. Nowadays it appears that although the calculated absolute risk values (especially among middle-aged patients) are often relatively low, the relative risk is unexpectedly high. Hence the idea of "car- diac age" or "coronary age" was born. It is still a relatively new concept. It seems that Grundy was the first to postulate in 1999 the use of biological age, based on evidence of arteriosclerosis, instead of the previously used chronological age, to estimate the cardiovascular risk by Framingham scale [1]. Although the advancement of atherosclerosis progresses with age, there is great individual variability at the onset and the speed of progression of atherosclerotic process. This may result in overestimation or underestimation of risk. That is why recently it has been postulated that arterial age be included in global risk scales.

Obesity is considered as a risk factor for the early development of atherosclerosis and cardiovascular disease.

Correspondence address:

Adam Lemanowicz, PhD, Department of Radiology and Diagnostic Imaging, Nicolaus Copernicus University in Toruń, Collegium Medicum in Bydgoszcz,

9 Skłodowskiej-Curie St., 85-094 Bydgoszzz, Poland, e-mail: adam.lemanowizz@gmail.com

Authors' contribution:

A Study design · B Data collection · C Statistical analysis · D Data interpretation · E Manuscript preparation · F Literature search · G Funds collection 
In recent studies not the total amount of body fat but rather its specific regional distribution is considered as a risk factors for cardiovascular disease. In this context, many studies have focused on intra-abdominal adipose tissue, which has features of an active endocrine gland and secretes a number of hormones and atherogenic cytokines known as adipokines [2]. Pericardial adipose tissue is considered a part of visceral fat, and it is believed that, in addition to systemic effects of adipokines, it can exert a local atherogenic effect on the coronary arteries [3]. Patients with morbid obesity are a special group of patients because they present multiple individual risk factors of atherosclerosis, some of which may enhance atherogenic effect due to interactions.

However, recently multiple reports have emerged, in which the direct association of obesity with the development of atherosclerosis is questioned. In some patients with obesity, and in some pathologies, an increased survival rate is observed compared to people with normal body mass. This phenomenon has been termed the "obesity paradox" or "reversed epidemiology". Such a paradoxical relationship of high body mass index (BMI) with better forecasts has already been described in patients with chronic renal disease, chronic heart failure, chronic obstructive pulmonary disease, and in patients with coronary heart disease [4]. This observation is explained by the fact that the nature and location of body fat, and not its total amount in the body, are risk factors for these diseases. Because subcutaneous adipose tissue is metabolically nearly inactive in comparison to visceral fat, its dominance in the body could actually explain the phenomenon of "reversed epidemiology". Recent studies have even shown that in healthy individuals, an increased amount of subcutaneous adipose tissue has a protective effect, inhibiting the development of atherosclerosis [5]. The phenomenon of the obesity paradox encourages further research, especially in the context of linking it with clusters of adipose tissue in particular areas of the body.

An aim of the study was to investigate the risk of CAD in morbidly obese patients using coronary artery calcium measurement.

\section{Material and methods}

In this prospective study we evaluated 51 subjects with morbid obesity, who were scheduled for bariatric surgery procedures in our hospital. Inclusion criteria were as follows: morbid obesity (BMI $\geq 40 \mathrm{~kg} / \mathrm{m}^{2}$ ) and lack of consistent effect of conservative treatment of obesity resulting in referral to bariatric surgery. Exclusion criteria included: body weight over $220 \mathrm{~kg}$ (scanner's table limit), inability to fit the scanner's gantry, dyspnoea in supine position, previous coronary stenting or coronary surgery, arrhythmia or tachycardia, pregnancy, and age below 18 years. Due to possible ethical concerns, a control group of 30 subjects was composed retrospectively of patients who were scanned due to clinical suspicion of coronary artery disease and who did not fulfil obesity criteria. Exclusion criteria were similar as in the study group. The study protocol was approved by a bioethical commission of our university, and all subjects gave their informed consent for participation in the study.

All subjects underwent an ECG-gated cardiac CT scanning according to the coronary calcium scoring protocol using a 64-row Philips Brilliance unit [6,7]. Acquisition of data was performed in $2.5-\mathrm{mm}$ slices at $120 \mathrm{kV}$ and $75 \mathrm{mAs}$, with an FOV of $220 \mathrm{~mm}$. No intravenous contrast media nor premedication were used. Standard Philips Brilliance Workstation with HeartBeat CS application were used to evaluate the presence and quantity of coronary artery calcium (CAC).

Measurement of the calcium score (CS) was carried out in a semi-automatic manner. In the initial stage the software distinguished calcifications on CT scans according to Agatston et al's criteria [8]. Then a reader was needed to identify coronary arteries at the axial cross-sections of the heart and to manually indicate highlighted calcifications. Other types of calcifications (such as calcified heart valves or picture noise erroneously classified by the algorithm as calcifications) were neglected by the reader. Then, the application automatically calculated Agatston's CS. Due to the high skewness of CS values, the data were log-transformed before statistical analysis [6].

Distribution of CS values within both groups was compared to the general population values using data from the German Multislice Normal Incidence of Coronary Health (MUNICH) Registry [9]. Apart from conventional CS, we also used coronary age (CA) as an alternative function of CAD risk. CA is defined as the age of a healthy person (without risk factors) who presents a risk of coronary heart disease equal to the risk calculated for the analysed patient. For example, if a coronary age of 65 years is calculated in 55-year-old patient, it means that the cardiovascular risk expressed by the amount of coronary calcifications is equal to that of a healthy 65-year-old individual. For the purpose of this study, patients' coronary age was determined by means of a commonly available on-line MESA-based tool [10,11].

Quantitative variables were expressed as mean with 95\% confidence intervals (95\% CI). Statistical analysis was performed using MedCalc v. 14 (MedCalc Software bvba, $\mathrm{NL}$ ). Normality of the data was tested using the Kolmogorov-Smirnov test. Differences in quantitative data were tested using the independent samples $t$-test. Comparison of qualitative data was performed using the Chi-squared test. $P$-values of 0.05 were considered statistically significant.

\section{Results}

The participants were aged between 19 and 71 years (mean 46.8 years). Cardiac CT scanning was completed 
Table 1. Demographic characteristics of the study groups. Mean values (95\% confidence interval) or structural indices and $p$-values for the differences are given

\begin{tabular}{|l|c|c|c|}
\hline Parameter & Study group & Control group & $p$ \\
\hline Gender (females) & $66.7 \%$ & $60.0 \%$ & 0.0663 \\
\hline Metrical age (years) & $44.1(40.9-47.3)$ & $51.8(47.9-55.7)$ & 0.0723 \\
\hline Body weight $[\mathrm{kg}]$ & $128.9(124.1-133.8)$ & $76.5(71.4-81.6)$ & 0.0000 \\
\hline Height $[\mathrm{m}]$ & $1.65(1.63-1.67)$ & $1.70(1.67-1.73)$ & 0.0024 \\
\hline BMl & $47.3(45.7-48.9)$ & $26.5(24.9-28.0)$ & 0.0000 \\
\hline Hypertension & $35 \%$ & $70 \%$ & 0.0023 \\
\hline Diabetes & $18 \%$ & $30 \%$ & 0.2113 \\
\hline Smoking & $16 \%$ & $43 \%$ & 0.0075 \\
\hline Dyslipidaemia & $22 \%$ & $47 \%$ & 0.0191 \\
\hline
\end{tabular}

Table 2. Results of measurement of coronary calcifications. Mean values ( $95 \%$ confidence interval) or structure index and significance level in $t$-test are given

\begin{tabular}{|l|c|c|c|}
\hline Parameter & Study group & Control group & $p$ \\
\hline Prevalence of calcifications & $52.9 \%$ & $23.3 \%$ & 0.0091 \\
\hline Calcium score & $91.9(0.0-189.4)$ & $45.6(8.2-83.0)$ & 0.0661 \\
\hline Coronary age [years] & $49.6(44.9-54.4)$ & $47.0(41.3-52.7)$ & 0.5057 \\
\hline Difference between coronary and metrical age [years] & $+5.55(1.51-9.59)$ & $-4.77(-10.02 ;-0.48)$ & 0.0023 \\
\hline
\end{tabular}

in all subjects without premedication. The mean heart rate during the acquisition was 73 BPM. Both groups statistically significantly differed regarding body weight, height, and BMI. Common risk factors for atherosclerosis, i.e. hypertension, diabetes, nicotine, and dyslipidaemia, were more frequent in the control group than in obese patients, and only in the case of diabetes was the difference not statistically significant. Detailed demographic characteristics of the study groups are given in Table 1 .

The prevalence of CAC was higher in morbidly obese patients than in controls $(p=0.0091)$ (Table 2$)$. In the obesity group, calcifications were detected in 27 individuals, and CS ranged from 1 to 2453 . A value greater than the $50^{\text {th }}$ centile was reached by $51 \%$ of subjects (Figure 1 ). The CA of patients ranged from 19 to 96 years, and $47 \%$ of the patients were above the metrical age (Figure 2). In the control group, calcifications were detected in seven subjects, and CS ranged from 1 to 346 . Only $23 \%$ of controls had CAC over the $50^{\text {th }}$ centile (Figure 1 ). The majority $(63 \%)$ of controls had CA lower than their metrical age (Figure 2).

The mean number of calcifications was higher in the study group than in the control group, but the difference was of borderline significance. However, the difference between coronary age and metrical age was significantly higher in obese patients than in controls $(p=0.0023)$ (Table 2). The ROC curve analysis confirmed that the difference between CA and metrical age presented the strongest relation with obesity (Table 3 ).

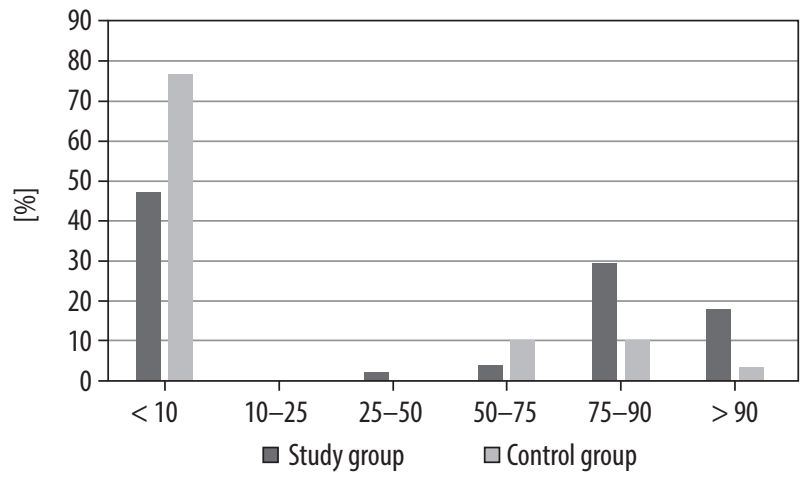

Figure 1. Percentile distribution of calcium score (CS) values in the study group and the control group, according to MUNICH registry

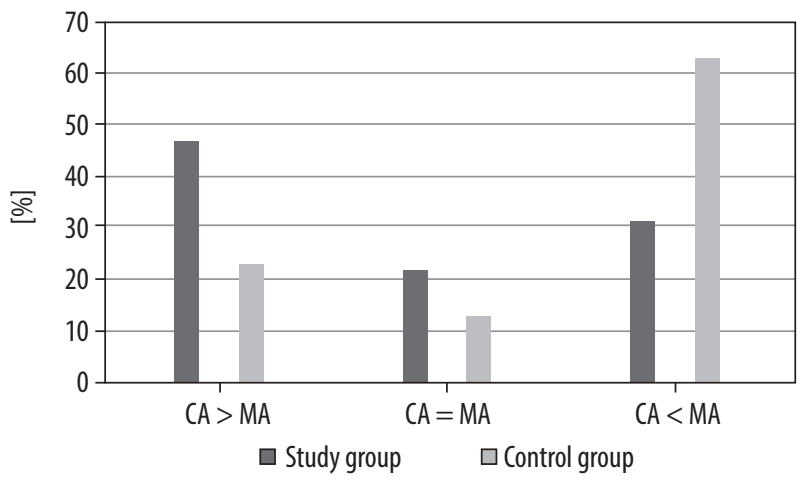

Figure 2. Distribution of the difference between coronary age $(C A)$ and metric age (MA) in the studied groups 
Table 3. Analysis of the ROC curve for the relationship between obesity and analysed morphological parameters. Only statistically significant relationships are shown

\begin{tabular}{|l|c|c|c|c|c|}
\hline Parameter & AUC $(95 \% \mathrm{Cl})$ & $p$ & Threshold & Sensitivity & Specificity \\
\hline Prevalence of coronary calcifications & $0.64(0.53-0.75)$ & 0.0069 & $>0$ & 52 & 77 \\
\hline Calcium score & $0.63(0.52-0.74)$ & 0.0302 & $>0.1$ & 58 & 77 \\
\hline Coronary age [years] & $0.71(0.60-0.81)$ & $<0.0001$ & $\leq 44$ & 58 & 90 \\
\hline Difference between coronary and metrical age [years] & $0.72(0.61-0.81)$ & 0.0005 & $>-6$ & 82 & 63 \\
\hline
\end{tabular}

\section{Discussion}

In our study we showed that patients with morbid obesity present an increased coronary age. In this study, the number of calcifications in the coronary arteries and derivative parameters were adopted as the primary indicators of risk for atherosclerosis. Our results indicate that obesity is associated with an increased risk of coronary atherosclerosis, which is reflected by a higher incidence of calcification in the study group compared to the control group. Interestingly, the difference between CA and metrical age presented the strongest relation to obesity. Therefore, it may be concluded that this parameter potentially may become a useful method for estimating the risk of cardiovascular events in people with extreme obesity. It also seems to be a unique finding because, according to our knowledge, the concept of the difference between coronary and metrical age has not been studied before.

Calcium scoring has been shown to be reproducible and predictive of a variety of adverse outcomes of CAD. Moreover, progression of calcifications correlates with severity of $\mathrm{CAD}$, which adds to the predictive value of this test $[9,10]$. Of course, coronary computed tomography angiography (CTA) would offer much more information on the status of coronary arteries than calcium scoring. However, the main drawbacks of CTA include much higher radiation dose and the risk of contrast-induced nephropathy (CIN) [12], which makes CTA inefficient as a screening tool. CIN is of particular importance because atherosclerosis remains an important risk factor of this pathology [12]. Nevertheless, little is known about the impact of obesity on the early development of atherosclerosis. The early studies, such as the Seven Countries Study (1984), revealed only a negligible correlation between body weight and the incidence of coronary heart disease. In the Pathological Determinants of Atherosclerosis in Youth (PDAY) study, BMI and subcutaneous fat thickness were positively correlated with the pathomorphological signs of early atherosclerosis in young men, but not in young women [13]. However, the large MESA study indicated that obesity is associated with an increased risk of atherosclerosis, which manifested, among others, by 1.2-fold higher incidence of calcification in the coronary arteries in obese patients compared with people of normal weight [14]. Today, the efforts of researchers tend to focus on determining the impact of the regional distribution of fat and subtypes of fat on the development of atherosclerosis. Among the available publications, particularly noteworthy is the work of Gadelha et al., who conducted their research on a population similar to ours, i.e. patients without known coronary artery disease, scheduled for bariatric surgery. The researchers noticed the presence of coronary CAC in $14 \%$ of participants, including $26 \%$ of men and $11 \%$ of women. They also underlined that the study participants with CAC were generally older (average age 46.8 years) than patients without calcifications in the coronary arteries (mean age 34.3 years). Other variables, showing the relationship with the CAC, included: male gender, type 2 diabetes, hypertension, and hypercholesterolaemia [15]. In our own material, calcifications in the coronary arteries in morbidly obese patients occurred much more frequently (52.9\%), wherein our patients were more obese than in the above-quoted paper (mean BMI was 47.3 vs. 40.5). Similarly, the results of the cohort, long-term CARDIA (Coronary Artery Risk Development in Young Adults) study suggested that abdominal obesity, assessed by waist circumference and waist-hip ratio (WHR) in young adults during a 15-year follow-up period, was directly linked with a higher incidence of coronary atherosclerosis [16]. Similar results were seen in the St. Francis Heart Study, in which abdominal obesity evaluated with WHR and amount of visceral fat positively correlated with the amount of CAC in people aged 50-70 years [17]. Meanwhile, Cassidy et al. analysed the coincidence of coronary calcifications with, for example, abdominal circumference, WHR, and BMI and discovered that expected annual growth of CAC in overweight people is about $51 \%$ higher than in people with underweight and normal weight [18]. In turn, the Muscatine Study demonstrated that excessive weight gain during childhood (8-18 years) and the calculated BMI in the age of 20-34 years were positively associated with the presence of calcifications in the coronary arteries later in life, i.e. in the age range 29-37 years [19].

The literature review gives grounds to believe that the vascular age indeed allows us to improve the efficiency of determining the cardiovascular risk. For example, Shaw et al. utilised CAC measurements performed in a group of 10,377 asymptomatic participants in order to assess the risk of death and then the number of life-years lost. Their results indicated a direct relationship between chronological age and the number of calcifications in the coronary 
arteries, and also allowed them to create their own linear predictive model. According to the researchers, CS below 10 in patients aged over 70 years was equivalent to a reduction in biological age by 10 years. In contrast, CS $>400$ in younger patients "added" up to 30 years of chronological age. In their material, the age modified in relation to the number of calcifications was also a better predictor of mortality than chronological age. With regard to the Framingham scale, utilisation of the modified age resulted in reclassification of patients from a low-risk to an intermediate risk group in $55 \%$ of cases, and $45 \%$ of those at medium risk to high risk [20]. Similar results were obtained by Nasir et al. In their material, CS ranging from the $75^{\text {th }}$ to the $90^{\text {th }}$ percentiles added 10 years to metrical age, while exceeding the $90^{\text {th }}$ percentile increased the age by 20 years. In contrast, in patients in age where the CS $>0$ falls within the normal range (i.e. in men above the age of 55 and in women above the age of 65 years), CS $=0$ reduced the age to 40-44 years and 55-59 years, respectively [21].

There are also limitations to our study that should be addressed. Firstly, our sample size was calculated with assumption for the largest possible effect. This might have led to the underestimation of the sample size to get statistical significance of the difference in the total CAC load. In fact, the difference in CS between the obesity group and control group is large and should be statistically significant when including a larger number of subjects. Secondly, selection of the control group should be discussed. Due to ethical concerns, exposition of healthy individuals to the radiation was not acceptable. Therefore, we used a group of subjects who were scheduled for a diagnostic coronary CT angiography. As a result, our control group participants might have more risk factors of atherosclerosis. To compensate for this we used centile ranks based on the MESA study as a reference. On the other hand, because our controls were slightly older than obesity patients, a direct comparison of CA showed a non-significant difference. Therefore, we used the difference between CA and metrical age as the method of coronary risk estimation. Finally, we used a CA estimation system based on the American population, which is more heterogeneous than the European one. However, this discrepancy might not limit the soundness of our conclusions because in general Americans tend to present no less risk of CAD than Europeans.

\section{Conclusions}

We found that morbid obesity is associated with an increased coronary age, which may reflect the raised cardiovascular risk in obese patients. However, further studies on larger populations and with follow-up are necessary to determine the actual usefulness of coronary age in the prediction of cardiovascular events.

\section{References}

1. Grundy SM. Age as a risk factor: you are as old as your arteries. Am J Cardiol 1999; 83: 1455-1457.

2. Hajer GR, Van Haeften TW, Visseren FL. Adipose tissue dysfunction in obesity, diabetes, and vascular diseases. Eur Heart J 2008; 29: 2959-2971.

3. Talman AH, Psaltis PJ, Cameron JD, et al. Epicardial adipose tissue: far more than a fat depot. Cardiovasc Diagn Ther 2014; 4: 416-429.

4. Niedziela J, Hudzik B, Niedziela N, et al. The obesity paradox in acute coronary syndrome: a meta-analysis. Eur J Epidemiol 2014; 29: 801-812.

5. Narumi H, Yoshida K, Hashimoto N, et al. Increased subcutaneous fat accumulation has a protective role against subclinical atherosclerosis in asymptomatic subjects undergoing general health screening. Int J Cardiol 2009; 135: 150-155.

6. Serafin Z, Lasek W, Laskowska K. Phantom-calibrated versus automatic coronary artery mass quantification with multidetector-row computed tomography: in vitro and in vivo study. Acta Radiol 2008; 49: 1007-1015.

7. Serafin Z, Rusak G, Strześniewski P, et al. Comparison of Calcium Scoring With 4-Multidetector Computed Tomography (4-MDCT) and 64-MDCT. J Comput Assist Tomogr 2012; 36: 88-93.

8. Agatston AS, Janowitz WR, Hildner FJ, et al. Quantification of coronary artery calcium using ultrafast computed tomography. J Am Coll Cardiol 1990; 15: 827-832.
9. Schmermund A, Erbel R, Silber S, MUNICH Registry Study Group. Multislice Normal Incidence of Coronary Health. Age and gender distribution of coronary artery calcium measured by four-slice computed tomography in 2,030 persons with no symptoms of coronary artery disease. Am J Cardiol 2002; 90: 168-173.

10. McClelland RL, Chung H, Detrano R, et al. Distribution of coronary artery calcium by race, gender, and age: Results from the Multi-Ethnic Study of Atherosclerosis (MESA). Circulation 2006; 113: 30-37.

11. The Multi-Ethnic Study of Atherosclerosis. Arterial Age Calculator. Available at: http://www.mesa-nhlbi.org/calcium/input.aspx

12. Serafin Z, Karolkiewicz M, Gruszka M, et al. High incidence of nephropathy in neurosurgical patients after intra-arterial administration of low-osmolar and iso-osmolar contrast media. Acta Radiol 2011; 52: 422-429.

13. McGill HC, McMahan CA, Herderick EE, et al. Obesity accelerates the progression of coronary atherosclerosis in young men. Circulation 2002; 105: 2712-2718.

14. Burke GL, Bertoni AG, Shea S, et al. The impact of obesity on cardiovascular disease risk factors and subclinical vascular disease: The multi-ethnic study of atherosclerosis. Arch Intern Med 2008; 168: 928-935.

15. Gadelha PS, Campos JM, Moraes F, et al. Altered coronary artery calcium scores before bariatric surgery. Springerplus 2014; 3: 199. 
16. Lee CD, Jacobs DR, Schreiner PJ, et al. Abdominal obesity and coronary artery calcification in young adults: the Coronary Artery Risk Development in Young Adults (CARDIA) Study. Am J Clin Nutr 2007; 86: 48-54.

17. Arad Y, Newstein D, Cadet F, et al. Association of multiple risk factors and insulin resistance with increased prevalence of asymptomatic coronary artery disease by an electron-beam computed tomographic study. Arter Thromb Vasc Biol 2001; 21: 2051-2058.

18. Cassidy AE, Bielak LF, Zhou Y, et al. Progression of subclinical coronary atherosclerosis: Does obesity make a difference? Circulation 2005; 111: 1877-1882.
19. Mahoney L, Burns T, Stanford W, et al. Coronary risk factors measured in childhood and young adult life are associated with coronary artery calcification in young adults: the Muscatine Study. J Am Coll Cardiol 1996; 27: 277-284.

20. Shaw LJ, Raggi P, Berman DS, Callister TQ. Coronary artery calcium as a measure of biologic age. Atherosclerosis 2006; 188: 112-119.

21. Nasir K, Vasamreddy C, Blumenthal RS, Rumberger J. Comprehensive coronary risk determination in primary prevention: an imaging and clinical based definition combining computed tomographic coronary artery calcium score and national cholesterol education program risk score. Int J Cardiol 2006; 110: 129-136. 